\title{
IDENTIFICAÇÃO DE EMBARCAÇÕES EM IMAGENS \\ AEROTRANSPORTADAS DE RADAR DE ABERTURA SINTÉTICA (R-99 SAR) NA ÁREA MARÍTIMA DO BRASIL
}

Identifying vessels from airborne synthetic aperture radar images (R-99 SAR) in the coastal area of Brazil

SÉRGIO ROBERTO HORST GAMBA ${ }^{1,2}$

EDSON EYJI SANO ${ }^{3}$

MARCELO PERES ROCHA ${ }^{2}$

${ }^{1}$ Comando-Geral de Operações Aéreas - COMGAR

Força Aérea Brasileira - FAB

SHIS QI-05 - Área Especial 12, CEP: 71615-600 Brasília - DF, Brasil

${ }^{2}$ Universidade de Brasília - UnB

Campus Universitário Darcy Ribeiro, CEP: 70910-900 Brasília - DF, Brasil

${ }^{3}$ Embrapa Cerrados

BR-020 km 18 CEP: 73301-970 Planaltina, DF, Brasil

horsthess@msn.com, sano@cpac.embrapa.br, peres_marcelo@yahoo.com.br

\section{RESUMO}

O objetivo deste trabalho foi identificar embarcações em imagens de radar obtidas pela aeronave R-99 da Força Aérea Brasileira. Dados de amplitude, obtidas na banda L e nas polarizações HH, HV, VH e VV da região de Porto de Tubarão, ES, foram processados por meio de diferentes tipos de realces, filtros, classificadores e transformadores espectrais. As imagens com maior potencial para identificar embarcações foram ainda analisadas para diferenciar embarcações militares de mercantes, considerando-se os cinco elementos de interpretação (forma, tamanho, sombra, tonalidade e fatores associados, isto é, o contexto em que as embarcações se encontram nas imagens) e as cinco fases de interpretação de imagens (detecção, reconhecimento, análise, dedução e classificação). A combinação de processamentos mais favoráveis foi o realce com contraste 50-200, seguido de filtro abertura ou erosão e classificador SVM (Support Vector Machine) ou transformação SCI (Synthetic Color Image). Foi possível discriminar embarcações nas fases de deteç̧ão e reconhecimento, enquanto a diferenciação entre 
embarcações mercantes e militares foi obtida nas fases de análise e dedução. No nível de classificação, não foi possível definir o tipo de embarcação militar (e.g., fragata ou contratorpedeiro) ou o tipo de embarcação mercante (e.g., petroleiro ou graneleiro).

Palavras-chave: Sensoriamento Remoto; Caracterização de Embarcações; Processamento de Imagens SAR; Realce Espectral.

\section{ABSTRACT}

The objective of this study was to identify vessels in radar images obtained by the R-99 airplane from Brazilian Air Force. Amplitude data obtained in L-band and HH-, HV-, VH- and VV-polarizations over the region of Tubarão Harbor, State of Espirito Santo, were processed through different types of enhancements, filters, classifiers, and spectral transformers. The images with highest performances were also analyzed to differentiate between military and merchant vessels, considering the five elements of interpretation (shape, size, shadow, tone and associated factors, that is, the context in which the vessels are found in the images) and five steps of image interpretation (detection, reconnaissance, analysis, deduction, and classification). The best combination of image processing was 50-200 contrast enhancement, aperture or erosion filters, and SVM (Spectral Vector Machine) classifier or SCI (Synthetic Color Image) transformation. It was possible to discriminate vessels in the steps of detection and reconnaissance, while the differentiation between military and merchant vessels was possible in the steps of analysis and deduction. In the final step of classification, it was not possible to define the types of military vessels (e.g., frigate or destroyer) neither the types of merchant vessels (e.g., petroleum tanker or bulk carrier).

Keywords: Remote Sensing; Characterization of Vesselso SAR Image Processing; Spectral Enhancement.

\section{INTRODUÇÃO}

No Brasil, o transporte marítimo é responsável por mais de $95 \%$ da movimentação de mercadorias relacionadas com comércio exterior. Em tempos de globalização, a fabricação de muitos produtos nacionais depende da importação de insumos, de tal sorte que interferências inapropriadas sobre o tráfego nos mares podem levar a nação ao colapso. Nesse sentido, é importante que o país conheça melhor e em maior detalhe, a sua Área Marítima. Nela, há a presença constante de embarcações responsáveis pela navegação de cabotagem (comércio entre portos nacionais) e de longo curso (comércio entre portos internacionais). Existe também a possibilidade de presença de navios militares estrangeiros que requerem monitoramento (VIDIGAL et al., 2006). Conforme destacado por Tello et al. (2006) e Paes et al. (2010), extensas Áreas Marítimas como é o caso da do Brasil ( 4,5 milhões de $\mathrm{km}^{2}$ ), necessitam de outras formas de vigilância que complementem as já existentes (reconhecimento visual e fotográfico). 
O uso de dados de sensoriamento remoto óptico para identificar embarcações é limitado por causa da impossibilidade de se adquirir imagens sob condições de cobertura de nuvens ou à noite. Além disso, a dificuldade aumenta quando a turbidez das águas é elevada (WU et al., 2009). Como conseqüência, dados de radar de abertura sintética (SAR) têm sido amplamente utilizados para identificar navios (e.g., ELDHUSET, 1996; WACKERMAN et al., 2001; TOUZI et al., 2004; LIU et al., 2005). Além das já bem conhecidas vantagens de se utilizar dados provenientes de radares imageadores, isto é, a sua capacidade de imageamento independente das condições atmosféricas e de iluminação solar (PARADELLA et al., 2005), a identificação de embarcações marítimas por meio de dados de radar é promissora porque elas agem como se fossem refletores de canto (alvos que refletem a maior parte da radiação incidente na faixa espectral de microondas, por meio de reflexão especular dupla) (HUANG et al., 2009). Neste caso, embarcações aparecem como manchas brilhantes em imagens de radar, independentemente se estão paradas ou em movimento.

No entanto, a capacidade de detecção de embarcações marítimas em imagens de radar depende de características como tamanho, forma e estrutura da embarcação, orientação em relação ao pulso de radar e estado do mar (MOUCHOT e GARELLO, 1998), além dos parâmetros de imageamento do sensor. De uma maneira geral, sensores com alta resolução espacial (tipicamente, 3-5 metros) apresentam maiores probabilidade de detecção, especialmente quando as dimensões das embarcações são reduzidas, porém, possuem a desvantagem de apresentar faixas de imageamento menores (CRISP, 2004).

Desde 2005, o Brasil possui sensores de radar embarcados nas aeronaves R-99 do Segundo Esquadrão do Sexto Grupo de Aviação (2/6 GAv) da Força Aérea Brasileira (R-99/SAR). Esses sensores operam nas bandas X e L (comprimentos de onda de $3 \mathrm{~cm}$ e $23 \mathrm{~cm}$, respectivamente) e com resoluções espaciais de 3 metros, 6 metros ou 18 metros (ALVES et al., 2009). Pesquisas com esses dados têm sido realizadas para identificar pistas de pouso (ALVES et al., 2009), discriminar culturas agrícolas (SILVA et al., 2009) e mapear incrementos de desmatamento na Amazônia (GUERRA et al., 2010). Nenhuma pesquisa foi ainda conduzida para identificar embarcações. O objetivo deste trabalho é identificar embarcações em imagens SAR do R-99 da área marítima do Brasil.

\section{METODOLOGIA}

Como área-teste, foi selecionada a região do Porto de Tubarão (20¹4’53” e

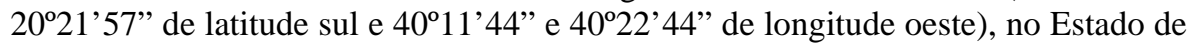
Espírito Santo, com comprovada concentração de embarcações mercantes e militares. O porto movimenta atualmente 65 milhões de toneladas de ferro e pellets (combustível sólido de resíduos de madeira prensado, proveniente de desperdícios de madeira) e 1,5 milhões de toneladas de grãos por ano (CODESA, 2010). 
Foram utilizadas imagens SAR em nível aerotransportado (plataforma R-99) com as seguintes características de imageamento: banda L, polarizações $\mathrm{HH}, \mathrm{VH}$, HV e VV, resolução azimutal de 3 metros, resolução em alcance de 3 metros, ângulo de incidência de $30^{\circ}$ data de passagem de 19 de março de 2008. As imagens em amplitude foram recebidas com resolução radiométrica de 8 bits e com correção radiométrica e geométrica, mas sem o conjunto completo de dados auxiliares necessários para a transformação em coeficientes de retroespalhamento. Essas imagens foram, em seguida, convertidas para o sistema de coordenadas geográficas (latitude e longitude) e datum WGS-84 e recortadas no tamanho aproximado de 8 km x 12 km.

O primeiro procedimento de análise dessas imagens foi feito por meio da extração de um conjunto de 50 amostras de valores digitais correspondentes a embarcações atracadas em cais (estruturas lineares onde as embarcações permanecem encostadas paralelamente) e piers (estruturas lineares estreitas perpendiculares ao cais) e de outro conjunto de 50 amostras correspondentes a instalações portuárias adjacentes. As amostras foram definidas visualmente no monitor do computador por meio da função de definição de regiões de interesse (ROI), disponível no aplicativo ENVI 4.3 ${ }^{\mathrm{TM}}$. Cada amostra foi formada por um número mínimo de 35 pixels e um número máximo de 56 pixels. Os dois conjuntos de dados foram analisados estatisticamente por meio do teste $t$ de Student.

\subsection{Discriminação de Embarcações em Imagens SAR}

Um conjunto de realces e classificações digitais de imagens foi testado com o intuito de definir a combinação de técnicas mais apropriadas para discriminar embarcações em imagens SAR (Figura 1). As imagens originais foram processadas por cinco técnicas de realce comumente utilizadas na literatura (RICHARDS e JIA, 2006; JENSEN, 2009): linear 2\%, linear 50-200, gaussiano, equalização de histogramas e raiz quadrada. Essas técnicas correspondem a uma transformação radiométrica pixel-a-pixel que aumenta a capacidade de discriminação visual de feições nas imagens com baixo contraste. Cada valor digital do pixel é modificado por uma transformação específica, sem levar em consideração os níveis de cinza dos pixels vizinhos. Essa operação é realizada através da manipulação do histograma da imagem, o qual descreve a distribuição estatística dos níveis de cinza da imagem em termos de freqüência de ocorrência de cada nível de cinza.

O realce linear $2 \%$ aplica um contraste linear com um corte de $2 \%$ nas duas extremidades do histograma, enquanto o realce linear 50-200 aplica um contraste linear assumindo 50 e 200 como valores de cortes inferior e superior, respectivamente. Nos dois casos, os valores definidos como cortes inferiores e superiores são reescalonados para 0 e 255, respectivamente. Os outros valores digitais dentro do intervalo são linearmente reescalonados entre 0 a 255. Nesse estudo, os valores 50 e 200 foram determinados empiricamente, após testes com diferentes intervalos. 
No realce gaussiano, os histogramas originais são alterados de modo a enquadrar os dados originais em uma distribuição normal, assumindo uma média de valores digitais igual a 127 e um intervalo de valores digitais de \pm 3 desvios-padrões que são realocados para valores 255 e 0, respectivamente.

Figura 1 - Fluxograma com as principais etapas do trabalho.

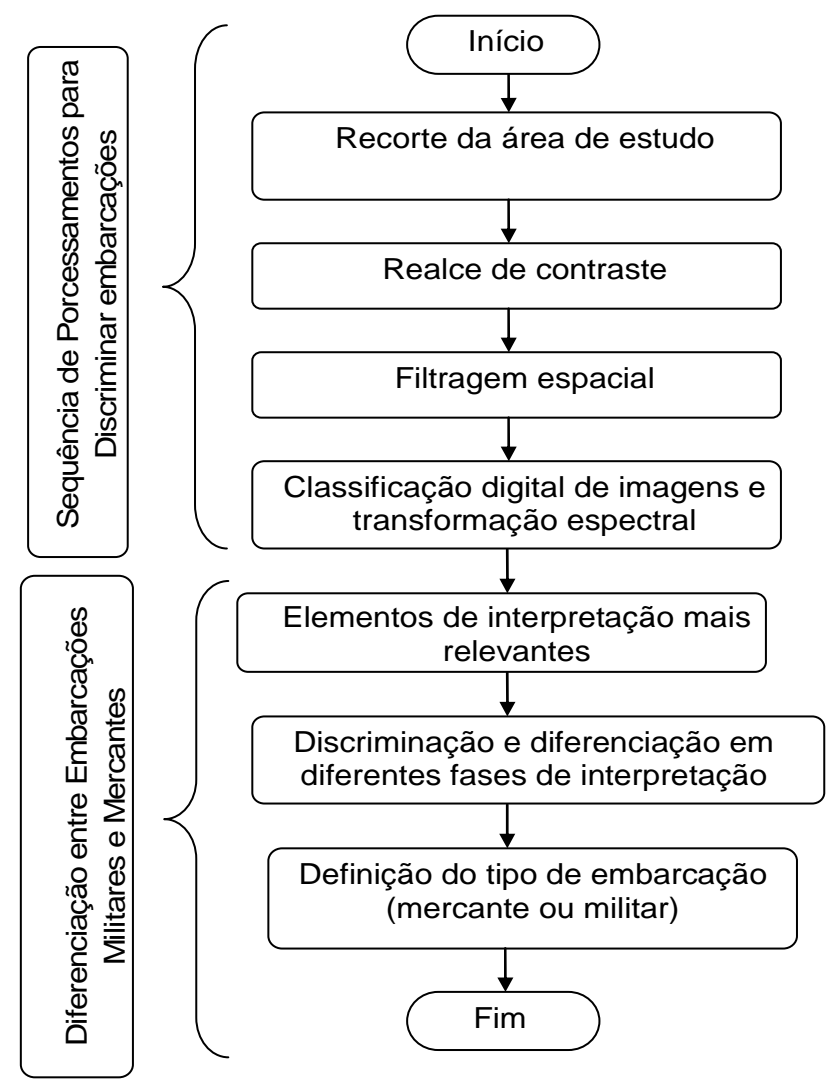

Na equalização, o histograma original é alterado para que o histograma resultante apresente barras com alturas aproximadamente iguais, isto é, os valores digitais originais são redistribuídos de tal forma que a freqüência de ocorrência de cada valor digital é aproximadamente igual. Como conseqüência, a equalização aumenta o realce de alvos com valores digitais medianos e reduz o contrate de alvos com valores digitais baixos e altos. A função raiz quadrada tenta melhorar o realce de alvos com tonalidades mais escuras na imagem, portanto, ela é apropriada 
quando o histograma original possui concentração excessiva de valores digitais menores.

O realce que apresentou melhor desempenho foi selecionado para a etapa seguinte de filtragem espacial. O critério utilizado para essa seleção foi baseado e adaptado da proposta do Freund e Simon (2000). Segundo esses autores, a imagem que apresenta o melhor contraste final é a que apresenta a menor média e o maior desvio-padrão. Para esse estudo, o critério utilizado foi a da menor média. Como a região de estudo apresenta a maior parte da área ocupada por oceano, a imagem que apresentar a menor média de valores digitais tende a apresentar um contrate melhor entre oceano e embarcação.

Os seguintes filtros espaciais morfológicos (HARALICK et al., 1987) foram aplicados: dilatação, erosão, abertura e fechamento. Os dois primeiros provocam efeitos de dilatação das partes escuras das imagens e efeitos de erosão das partes claras das imagens, respectivamente. Filtros de abertura envolvem o encadeamento do filtro de erosão, seguido de dilatação. Filtros de fechamento envolvem o encadeamento do filtro de dilatação, seguido de erosão. Filtros espaciais são úteis para destacar bordas, feições lineares dispostas em determinadas direções e padrões de textura, através do realce ou da supressão de determinadas freqüências espaciais (RICHARDS e JIA, 2006). Novamente, aquelas imagens filtradas com melhor realce, i.e., menor média de valores digitais foram selecionadas para as etapas posteriores de classificação digital e transformação espectral.

Os classificadores não-supervisionados testados foram o Isodata e o K-médias, enquanto os classificadores supervisionados analisados foram: Distância Mínima, Distância Mahalanobis, Máxima Verossimilhança (MAXVER), Spectral Angle Mapper (SAM), Spectral Information Divergence (SID), codificação binária e Support Vector Machine (SVM). A classificação de imagens consiste em associar um rótulo para cada pixel da imagem. Os níveis de cinza associados a cada pixel são identificados, por exemplo, por meio de classes de cobertura das terras (água, vegetação, solo, rocha, etc.) (SCHOWENGERDT, 2007). Para a classificação supervisionada, foram extraídas amostras de treinamento de valores digitais em áreas representativas de embarcações, instalações do cais, oceano, vegetação e solo. O total de pixels variou de 4.227 pixels (embarcação) a 144.157 pixels (oceano). Testes foram ainda realizados com classificações envolvendo três dessas cinco classes (embarcações, instalações do cais e água).

O desempenho dos classificadores foi avaliado pelo coeficiente Kappa (K) (LANDIS e KOCH, 1977). A equação que fornece o valor de $K$ é:

$$
K=\frac{N \sum_{i=1}^{Y} X_{i i}-\sum_{i=1}^{Y} X_{i+} X_{+i}}{N^{2}-\sum_{i=1}^{Y} X_{i+} X_{+i}}
$$


onde $\gamma$ é o número de linhas e de colunas e $N$ é o número total de pontos.

Em termos de transformadores espectrais, as seguintes técnicas foram aplicadas: decorrelação, saturação e Synthetic Color Image (SCI). A escolha da melhor imagem classificada foi baseada na matriz de confusão (LANDIS e KOCH, 1977), enquanto que a melhor imagem transformada foi baseada na maior média. Foram utilizadas as três polarizações com as menores correlações de Pearson.

\subsection{Identificação do Tipo de Embarcação (Mercante ou Militar)}

As imagens classificadas e transformadas com melhor desempenho para discriminar embarcações foram analisadas visualmente para verificar os seus potenciais para diferenciar as embarcações em militar ou mercante. As embarcações militares possuem formato hidrodinâmico, necessário para o desenvolvimento de velocidades mais altas (Figura 2). No convés, são encontrados dispositivos como armamentos, equipamentos eletrônicos e superestrutura no seu centro. Os navios mercantes possuem cascos retangulares, com proa mais arredondada. No convés, são encontrados mastros, paus-de-carga, contêineres e superestrutura na metade traseira da embarcação (BRASIL, 2000).

Figura 2 - Vista de cima de embarcações militares e mercantes típicas.

Fonte: BRASIL (2000).

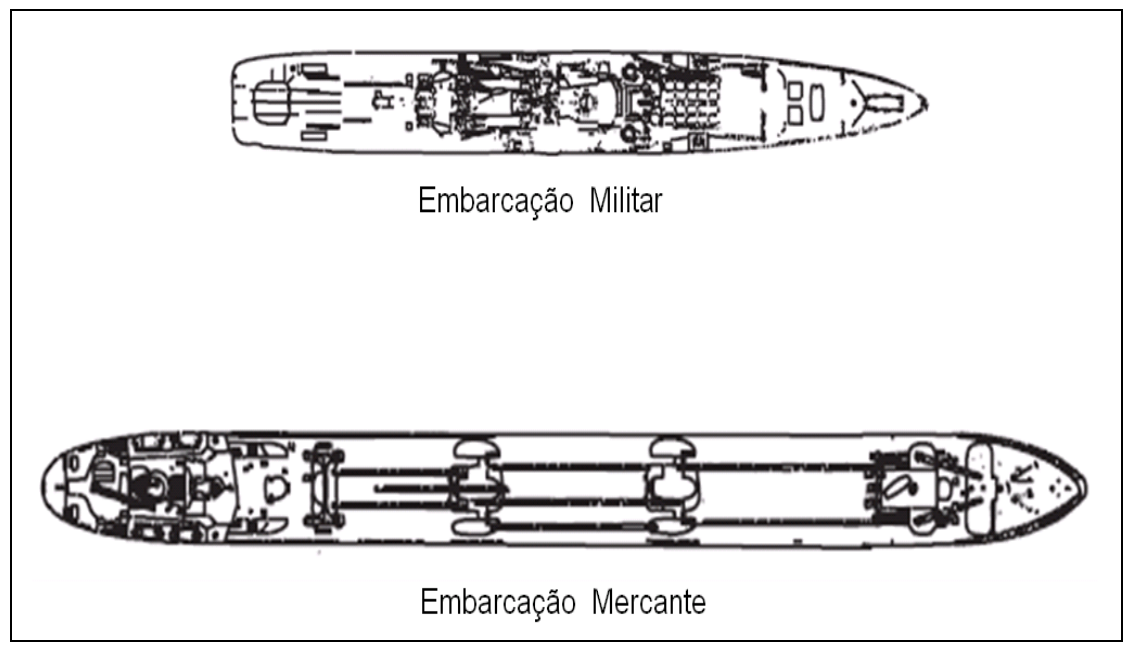

Bol. Ciênc. Geod., sec. Artigos, Curitiba, v. 17, no 3, p.456-475, jul-set, 2011. 
Forma, tamanho, sombra, tonalidade e fatores associados (contexto em que os objetos se encontram nas imagens) de embarcações militares e mercantes presentes nas imagens processadas nas etapas anteriores desse estudo foram analisados nas diferentes fases de interpretação visual de imagens. A definição da forma de um alvo em uma imagem SAR depende da geometria do imageamento do sistema SAR (resolução espacial, distorção de escala na direção de visada, deslocamento devido ao relevo e distorções causadas pelo movimento da plataforma). O tamanho que um alvo apresenta numa imagem depende da resolução espacial do sistema, enquanto o sombreamento está relacionamento com o ângulo de incidência e orientação alvoplataforma. Tonalidade é dependente do comprimento de onda, da polarização e do ângulo de incidência do sistema sensor, além das características dos alvos, principalmente da rugosidade, da constante dielétrica e da estrutura do alvo.

As fases consideradas foram a detecção, o reconhecimento, a análise, a dedução e a classificação (MECOCCI et al., 1995; ELDHUSET, 1996). A detecção tem relação direta com a visibilidade dos alvos. Embarcações podem ser visualizadas ou não em imagens SAR, dependendo de fatores como qualidade das imagens, resolução espacial do sensor e contraste entre o alvo e o meio (DAMIÃO et al., 2001). O reconhecimento estabelece “o que é o alvo” (por exemplo, aeronave, ponte, embarcação ou antena). O reconhecimento de alvos em imagens SAR é feito principalmente por meio da observação da forma e da tonalidade.

A análise é o estágio onde o alvo de interesse deve ser observado em detalhes. Por exemplo, pulsos de radar que incidem sobre as estruturas físicas de duas e três faces do convés geralmente geram borrões nas imagens SAR, dificultando a diferenciação dos componentes presentes no convés. Este problema torna-se ainda mais acentuado em embarcações militares, por causa da presença de armamentos, equipamentos eletrônicos e estruturas físicas de vários formatos.

A dedução é o estágio mais complexo, pois se fundamenta nos fatores associados ou convergência de evidências. O alvo é observado não isoladamente, mas como parte de todo um contexto (DAMIÃO et al., 2001). Por último, classificação é o estágio das conclusões em que se chega à compreensão da identidade e do verdadeiro significado do alvo.

Para identificar as embarcações encontradas na região do Porto de Tubarão em mercantes ou militares, foram adotados os seguintes critérios visuais: I = insuficiente, isto é, não atende às definições de forma, tamanho, sombra, tonalidade e fatores associados; e $\mathrm{S}=$ satisfatória, isto é, atende as definições dos cinco elementos de interpretação.

\section{RESULTADOS}

Em todas as três polarizações analisadas ( $\mathrm{HH}, \mathrm{VV}$ e $\mathrm{VH})$, os valores digitais correspondentes a embarcações e instalações portuárias adjacentes situaram-se próximos do nível de saturação, isto é, muito próximos do valor 255 (Tabela 1). De certa forma, este fato já era esperado por causa da reflexão especular dupla 
(refletores de canto) mencionada na parte introdutória deste texto. A análise estatística pelo teste $t$ de Student mostrou que é possível diferenciar embarcações de instalações portuárias nas três polarizações consideradas (rejeição da hipótese Ho, Tabela 1) e que a polarização VV possui maior potencial de discriminação [maior diferença entre $t_{\text {crit }}\left(t\right.$ crítico) e $t_{\text {cal }}(t$ calculado)].

\subsection{Discriminação de Embarcações em Imagens SAR}

Na Figura 3, são mostradas imagens SAR (banda L, polarização HH) da região de Porto de Tubarão, realçadas simplesmente pelos contrastes linear 2\%, linear 50200, gaussiano, equalização de histogramas e raiz quadrada. Os valores médios de amplitude e os correspondentes desvios-padrões dessas imagens realçadas são mostrados na Tabela 2. De acordo com o critério de menor média de valores digitais, mencionado anteriormente, as cenas que apresentaram os melhores realces foram aqueles processados com contraste linear 50-200.

Na Figura 4, são mostradas imagens SAR (banda L e polarização $\mathrm{HH}$ ) da mesma região de Porto de Tubarão e realçadas pelo contraste 50-200 e por quatro filtros espaciais distintos. As médias dos valores digitais e os correspondentes desvios-padrões dessas imagens realçadas são mostrados na Tabela 3. De acordo com as informações constantes desta tabela, os filtros abertura e erosão foram os que apresentaram melhores desempenhos.

Tabela 1. Análise da separabilidade espectral entre embarcações (EMB) e instalações portuárias adjacentes (INS) por meio de teste $t$ de Student, presumindo amostras de valores digitais com variâncias diferentes e nível de significância $\alpha=$ 0,05 .

\begin{tabular}{l|c|c|c}
\hline \multicolumn{1}{c|}{ Parâmetro } & \multicolumn{3}{|c}{ Polarização } \\
\cline { 2 - 4 } \multicolumn{1}{c|}{ Estatístico } & HH & VV & HV \\
\hline Média & 254 (EMB) & 253 (EMB) & 250 (EMB) \\
& 251 (INS) & 236 (INS) & 236 (INS) \\
\hline Variância & 3 (EMB) & 22 (EMB) & 197 (EMB) \\
& 42 (INS) & 333 (INS) & 309 (INS) \\
\hline Grau de liberdade & 55 & 55 & 93 \\
\hline$t_{\text {cal }}$ & 3,276 & 6,082 & 4,514 \\
\hline$t_{\text {crit }}$ & 2,004 & 2,004 & 1,986 \\
\hline Hipotese Ho & Rejeita & Rejeita & Rejeita \\
\hline
\end{tabular}

$t_{\text {cal }}=t$ calculado; $t_{\text {crit }}=t$ crítico. 
Figura 3 - Imagens SAR na banda $\mathrm{L}_{\mathrm{HH}}$ da região de Porto de Tubarão, realçadas com contraste linear $2 \%$ (a), contraste gaussiano (b), equalização de histogramas (c), raiz quadrada (d) e contraste linear 50-200 (e).

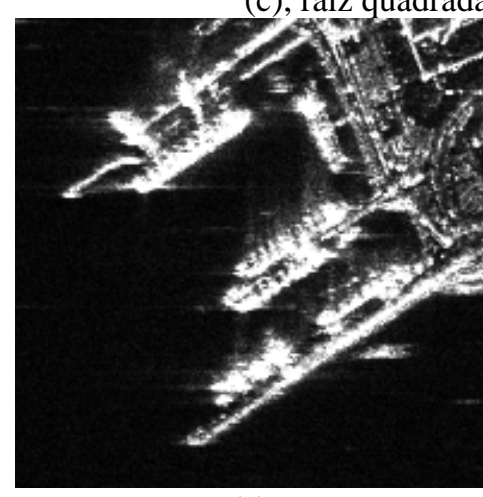

(a)

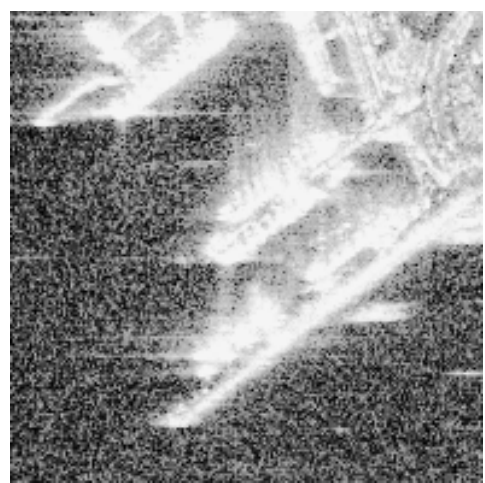

(c)

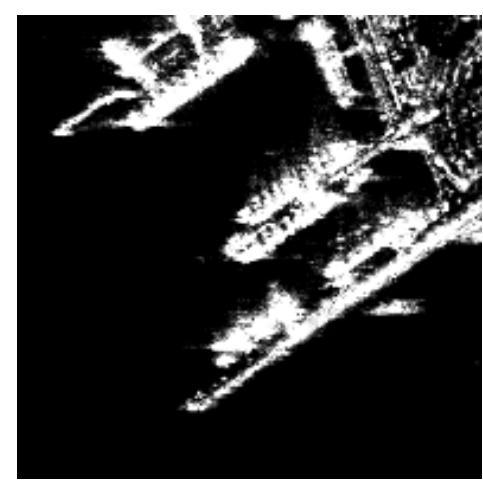

(e)

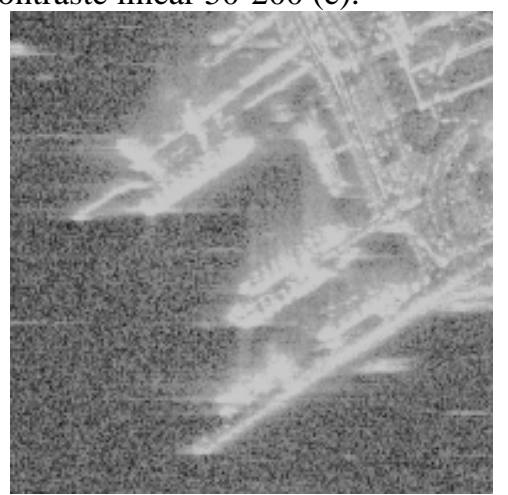

(b)

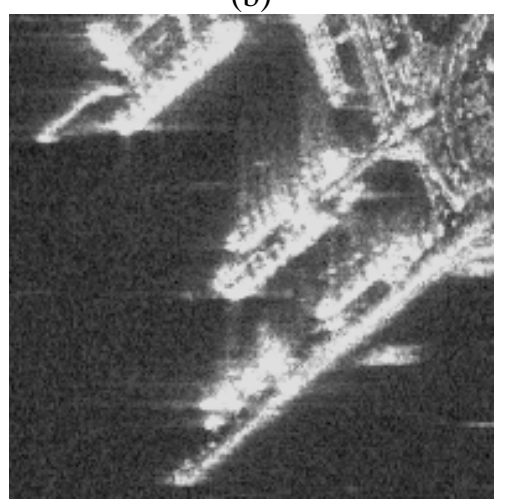

(d)

Bol. Ciênc. Geod., sec. Artigos, Curitiba, v. 17, no 3, p.458-475, jul-set, 2011. 
Tabela 2 - Médias de valores digitais de imagens SAR (banda L, polarizações $\mathrm{HH}$, HV, VH e VV) e correspondentes desvios-padrões (valores entre parênteses) da região de Porto de Tubarão, realçadas pelas técnicas de contraste linear 2\%, gaussiano, equalização de histogramas, raiz quadrada e linear 50-200.

\begin{tabular}{c|c|c|c|c}
\hline \multirow{2}{*}{ Contraste } & \multicolumn{4}{|c}{ Polarização } \\
\cline { 2 - 5 } & HH & HV & VH & VV \\
\hline Linear 2\% & 49,74 & 51,08 & 50,64 & 59,46 \\
& $(67,29)$ & $(60,27)$ & $(59,22)$ & $(58,35)$ \\
\hline Linear 50-200 & 39,50 & 28,01 & 27,81 & 23,63 \\
& $(79,78)$ & $(60,59)$ & $(60,41)$ & $(53,72)$ \\
\hline Equalização de & 129,68 & 68,30 & 68,07 & 82,13 \\
histogramas & $(77,62)$ & $(74,48)$ & $(73,31)$ & $(67,94)$ \\
\hline Raiz quadrada & 88,83 & 89,64 & 87,52 & 92,83 \\
& $(68,29)$ & $(54,68)$ & $(55,59)$ & $(48,15)$ \\
\hline & & & & \\
\hline
\end{tabular}

Figura 4 - Imagens SAR realçadas com contraste linear 50-200 na banda $\mathrm{L}_{\mathrm{HH}}$ da região de Porto de Tubarão, processadas com quatro filtros morfológicos distintos: abertura (a), dilatação (b), erosão (c) e fechamento (d).

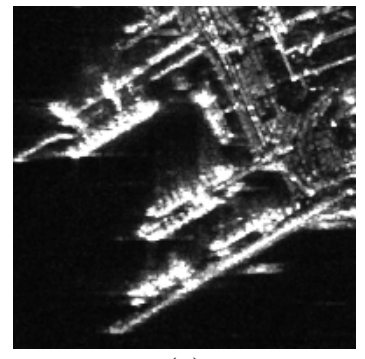

(a)

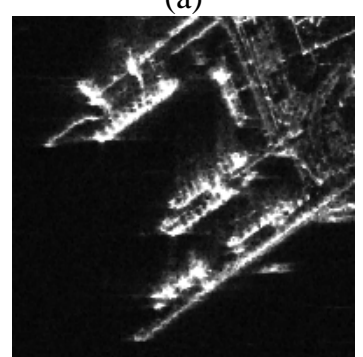

(c)

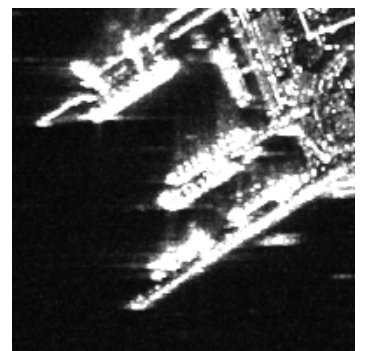

(b)

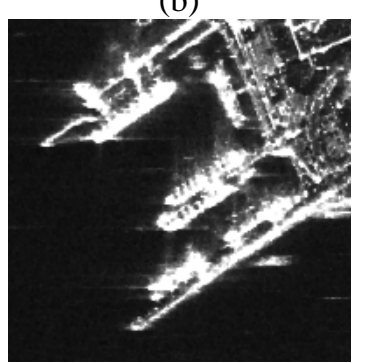

(d)

Tabela 3 - Médias de valores digitais de imagens SAR (banda L, polarizações HH, 
HV, VH e VV) e correspondentes desvios-padrões (valores entre parênteses) da região de Porto de Tubarão, realçadas pelas técnicas de contraste linear 50-200 e filtros espaciais abertura, dilatação, erosão e fechamento.

\begin{tabular}{c|c|c|c|c}
\hline \multirow{2}{*}{ Polarização } & \multicolumn{4}{|c}{ Filtros Espaciais } \\
\cline { 2 - 5 } & Abertura & Dilatação & Erosão & Fechamento \\
\hline HH & $48,03(74,20)$ & $70,48(91,88)$ & $36,71(63,46)$ & $59,52(83,96)$ \\
\hline HV & $49,24(70,60)$ & $75,28(91,72)$ & $37,69(59,98)$ & $63,20(82,55)$ \\
\hline VH & $49,35(70,09)$ & $75,50(91,01)$ & $37,79(59,53)$ & $63,34(81,82)$ \\
\hline VV & $54,98(71,46)$ & $87,77(89,95)$ & $40,90(60,14)$ & $72,64(81,01)$ \\
\hline
\end{tabular}

Na Tabela 4, são mostrados os coeficientes de correlação entre as quatro polarizações, a um nível de significância de 1\%. Polarizações com baixa correlação sugerem que as mesmas são complementares, isto é, informações que são realçadas em uma polarização não são em outra e vice-versa (ROSA, 1995). Resultados dessa tabela indicam uma alta correlação entre as polarizações cruzadas $\mathrm{HV}$ e VH, denotando redundância de informações. Dessa maneira, foi selecionada a combinação das polarizações HH, VV e VH para efetuar as etapas seguintes de classificação digital e transformação espectral.

Tabela 4 - Coeficientes de correlação de Pearson para as imagens SAR de Porto de Tubarão (banda L, polarizações HH, HV, VH e VV), realçadas com contraste linear 50-200 e filtro abertura.

\begin{tabular}{c|c|c|c|c}
\hline & $\mathrm{L}_{\mathrm{VH}}$ & $\mathrm{L}_{\mathrm{HV}}$ & $\mathrm{L}_{\mathrm{VV}}$ & $\mathrm{L}_{\mathrm{HH}}$ \\
\hline $\mathrm{L}_{\mathrm{VH}}$ & 1,000 & & & \\
\hline $\mathrm{L}_{\mathrm{HV}}$ & 0,992 & 1,000 & & \\
\hline $\mathrm{L}_{\mathrm{VV}}$ & 0,746 & 0,707 & 1,000 & \\
\hline $\mathrm{L}_{\mathrm{HH}}$ & 0,490 & 0,552 & 0,378 & 1,000 \\
\hline
\end{tabular}

Na Tabela 5, são mostrados os desempenhos dos classificadores supervisionado e não-supervisionado, considerando-se os valores de índice Kappa e de exatidão global. Baseados nesses valores, pode-se afirmar que o classificador SVM (Figura 5) apresentou o melhor desempenho. 
Tabela 5 - Índices Kappa e exatidões globais das imagens classificadas de Porto de

\begin{tabular}{l|c|c}
\multicolumn{3}{|c}{ Tubarão. } \\
\hline Classificador & Índice Kappa & Exatidão Global (\%) \\
\hline Isodata* & $-0,041$ & 8,03 \\
\hline K Médias* & $-0,041$ & 8,03 \\
\hline Distância Mínima** $^{*}$ & 0,691 & 82,10 \\
\hline Distância Mahalanobis** & 0,680 & 81,17 \\
\hline Máxima Verossimilhança** & 0,753 & 85,51 \\
\hline Spectral Angle Mapper (SAM)** & 0,049 & 8,97 \\
\hline $\begin{array}{l}\text { Divergência de Informação } \\
\text { Espectral** }\end{array}$ & 0,160 & 36,81 \\
\hline Codificação Binária** & 0,000 & 0,00 \\
\hline Support Vector Machine (SVM)* & 0,905 & 95,44 \\
\hline
\end{tabular}

* três classes (embarcações, instalações do cais e água) ** cinco classes (embarcações, instalações do cais, água, vegetação e solo).

Figura 5 - Resultado da classificação pelo método SVM envolvendo as imagens SAR (banda L, polarizações HH, VH e VV) da região do Porto de Tubarão. Azul = mar; amarelo = instalações do cais; e vermelho = embarcações.

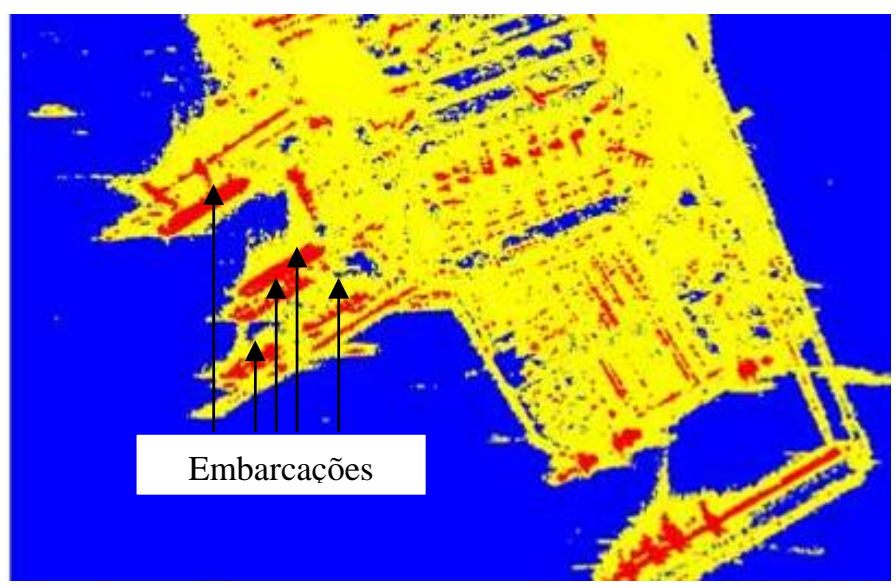

Bol. Ciênc. Geod., sec. Artigos, Curitiba, v. 17, no 3, p.456-475, jul-set, 2011. 
Com relação aos transformadores espectrais, considerando-se os resultados estatísticos da Tabela 6, verifica-se que a transformação SCI (maior média de valores digitais) é a que permite uma melhor discriminação das embarcações (Figura 6).

Tabela 6 - Médias e desvios-padrões das imagens SAR (banda L, polarizações HH, VH e VV) da região de Porto de Tubarão, realçadas por transformação linear 50-

200, filtro abertura e transformação espectral por decorrelação, saturação e Synthetic Color Image (SCI).

\begin{tabular}{c|c|c}
\hline Transformação Espectral & Média & Desvio-Padrão \\
\hline Decorrelação & 52,32 & 1,00 \\
\hline Saturação & 69,78 & 66,78 \\
\hline Synthetic Color Image (SCI) & 109,34 & 27,37 \\
\hline
\end{tabular}

Figura 6 - Imagens SAR (banda L, polarizações HH, VH e VV) da região do Porto de Tubarão, realçadas pelo contraste linear 50-200, filtro abertura e transformação espectral Synthetic Color Image (SCI).

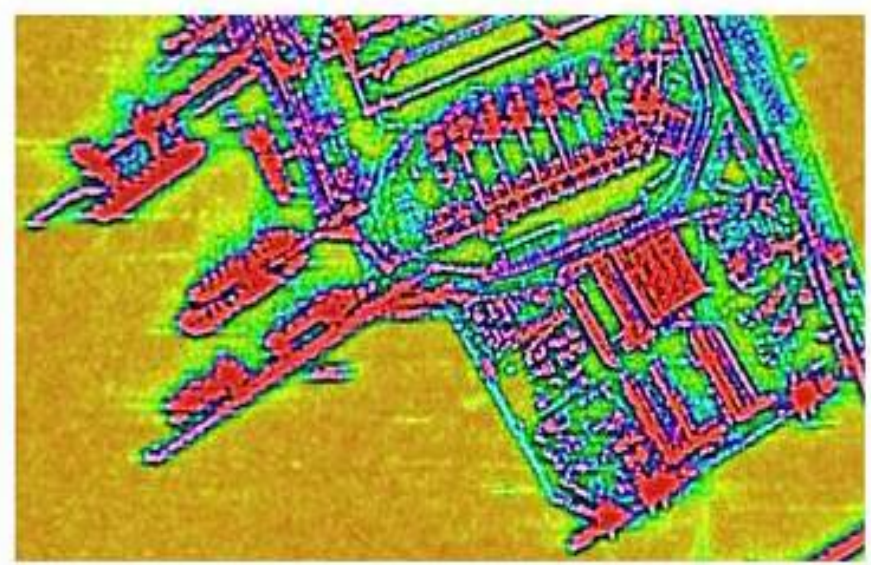

\subsection{Identificação de Tipo de Embarcação (Mercante ou Militar)}

Em ambas as imagens processadas por classificação SVM e por transformação SCI, a forma, o tamanho e a tonalidade foram os principais elementos de interpretação das imagens SAR que auxiliaram na diferenciação entre embarcações militares e mercantes (Tabela 7). Os elementos sombra e fatores associados ficaram no nível insuficiente.

Nas fases de detecção e reconhecimento, foi possível visualizar embarcações com o auxílio da forma, tamanho e tonalidade, porém, não foi possível diferenciar 
embarcações militares de mercantes (Tabela 8). Nas fases de análise e dedução, foi possível obter tal diferenciação com os elementos forma + tamanho e forma + tamanho + tonalidade, respectivamente. Na fase de classificação, nenhum elemento de interpretação foi aceitável para diferenciar embarcações.

Tabela 7 - Desempenho dos diferentes elementos de interpretação na diferenciação entre embarcações militares e mercantes presentes na região de Porto de Tubarão,

ES. S = satisfatório; $\mathrm{I}=$ insuficiente.

\begin{tabular}{|c|c|c|}
\hline \multirow{2}{*}{$\begin{array}{l}\text { Elemento de } \\
\text { Interpretação }\end{array}$} & \multicolumn{2}{|c|}{ Processamento de Imagens } \\
\hline & $\begin{array}{c}\text { Classificação SVM } \\
\text { (banda L, polarizações } \\
\text { HH, VV, VH) }\end{array}$ & $\begin{array}{c}\text { Transformador SCI } \\
\text { (banda L, polarizações } \\
\text { HH, HV, VH) }\end{array}$ \\
\hline Forma & $\mathrm{S}$ & $\mathrm{S}$ \\
\hline Tamanho & $\mathrm{S}$ & $\mathrm{S}$ \\
\hline Sombra & I & I \\
\hline Tonalidade & $\mathrm{S}$ & $\mathrm{S}$ \\
\hline Textura & I & $\mathrm{I}$ \\
\hline Padrão & $\mathrm{I}$ & $\mathrm{I}$ \\
\hline Densidade & I & I \\
\hline Declividade & I & I \\
\hline Posição & I & I \\
\hline
\end{tabular}

Tabela 8 - Nível de diferenciação entre embarcações militares e mercantes da região de Porto de Tubarão, ES em diferentes fases de interpretação de imagens baseados nos seguintes elementos de interpretação de imagens: forma, tamanho e tonalidade. $\mathrm{Dt}$ = detecção; $\mathrm{R}=$ reconhecimento; $\mathrm{A}$ = análise; $\mathrm{Dd}=$ dedução; $\mathrm{C}$ = classificação. $\mathrm{S}$ = satisfatória; $\mathrm{I}=$ insuficiente.

\begin{tabular}{c|c|c|c|c|c|c}
\hline \multirow{2}{*}{ Processamento } & Elemento de & \multicolumn{5}{|c}{ Fases de Interpretação } \\
\cline { 3 - 8 } & Interpretação & Dt & R & A & Dd & C \\
\hline \multirow{2}{*}{$\begin{array}{c}\text { Classificação SVM } \\
\text { (banda L, polarizações } \\
\text { HH, VV, VH) }\end{array}$} & Forma & S & S & S & S & I \\
\cline { 2 - 8 } & Tamanho & S & S & S & S & I \\
\cline { 2 - 7 } & Tonalidade & S & S & I & S & I \\
\hline $\begin{array}{c}\text { Transformador SCI } \\
\text { (banda L, polarizações } \\
\text { HH, HV, VH) }\end{array}$ & Forma & S & S & S & S & I \\
\cline { 2 - 7 } & Tamanho & S & S & S & S & I \\
\cline { 2 - 7 } & Tonalidade & S & S & I & S & I \\
\hline
\end{tabular}


O fator tamanho foi decisivo na diferenciação entre embarcações militares e mercantes. Conforme mostrado anteriormente, as embarcações mercantes são maiores que as militares, exceção os navios-aeródromos. Nas embarcações mercantes, através da forma e do tamanho, foi possível identificar detalhes presentes nos convés como a super-estrutura na parte traseira da embarcação, os mastros na extremidade dianteira da embarcação e estruturas no meio embarcação. A dedução foi uma das fases mais complexas de ser analisada, pois ela se fundamenta nos fatores associados ou convergência de evidências. Conforme mencionado anteriormente, o alvo é observado não mais de forma isolada, mas como parte de todo um contexto (DAMIÃO et al., 2001). Como as embarcações estavam atracadas próximos aos cais e piers de um porto civil, deduziu-se que se tratava de embarcações mercantes. Se as embarcações estivessem, por exemplo, atracados no porto da Baía de Guanabara, que pertence à Marinha, a dedução seria que se trataria de embarcações militares.

As imagens SAR do R-99 não forneceram detalhes suficientes para a última fase de interpretação, ou seja, a classificação. Nas imagens SAR analisadas nesse estudo, não foi possível definir, com precisão, o tipo de embarcação militar (e.g., fragata, contratorpedeiro ou navio-aeródromo) ou o tipo de embarcação mercante (e.g., petroleiro, carga-geral ou graneleiro). Esse tipo de identificação pode ser feito com imagens ópticas de alta resolução espacial.

\section{CONSIDERAÇÕES FINAIS}

Para discriminar embarcações em imagens SAR do R-99, a seqüência de processamento que apresentou os melhores resultados foi: realce de contraste 50200; filtragem abertura ou erosão; composição colorida envolvendo as polarizações $\mathrm{HH}$, VV e HV ou VH; e classificador SVM ou transformador SCI. Os elementos de interpretação passíveis de serem utilizados para a referida discriminação foram a forma, o tamanho e a tonalidade.

Dentre as cinco fases de interpretação (detecção, reconhecimento, análise, dedução e classificação), foi possível discriminar embarcações nas fases de detecção e reconhecimento. A diferenciação entre embarcações mercantes e militares foi possível nas fases de análise e dedução. No nível de classificação, nenhum elemento de interpretação foi aceitável para identificar o tipo de embarcação militar ou o tipo de embarcação mercante.

\section{AGRADECIMENTOS:}

Ao Comando Geral de Operações Aéreas (COMGAR), Força Aérea Brasileira (FAB), pela disponibilização das imagens SAR. 


\section{REFERÊNCIAS BIBLIOGRÁFICAS}

ALVES, S. A. S.; ASSANTA, L. R.; SANO, E. E.; MENESES, P. R. Abordagem metodológica baseada em imagens do SAR-R99B para identificar prováveis pistas de pouso não-homologadas na Amazônia. Acta Amazonica, v. 39, n. 3, p. 723-726, 2009.

BRASIL. Comando da Aeronáutica. Embarcações. Brasília, DF: [s.n.], 2000.

CODESA. Companhia Docas do Espírito Santo. Vitória, Espírito Santo, 2007. Disponível em: <http://www.portodevitoria.com.br>. Acesso em: 01 fev. 2010.

CRISP, D. J. The state-of-the-art in ship detection in synthetic aperture radar imagery. Edinburg, Austrália, DSTO Information Sciences Laboratory, 2004, 115 p. (Relatório DSTO-RR-0272).

DAMIÃO, D. P.; MENDES, V. L.; ROCHA, A.; FERNANDES, F. T.; BARROS, F. M. Sensoriamento Remoto Fotointerpretação II. São José dos Campos: Instituto de Estudos Avançados. [s.n.], 2001.

ELDHUSET, K. An automatic ship and ship wake detection system for spaceborne SAR images in coastal regions. IEEE Transactions on Geoscience and Remote Sensing, v. 34, n. 4, p. 1010-1019, 1996.

FREUND, J. E.; SIMON, G. A. Estatística Aplicada. Porto Alegre: Editora Bookman, 2000.

GUERRA, J. B.; MURA, J. C.; FREITAS, C. C. Discriminação de incrementos de desflorestamento na Amazônia com dados SAR R99B em banda L. Acta Amazonica, v. 40, n.3, p. 557-566, 2010.

HARALICK, R. M.; STENBERG, S. R.; ZHUANG, X. Image analysis using mathematical morphology. IEEE Transactions on Pattern Analysis and Machine Intelligence, v. 9, n. 4, p. 532-550, 1987.

HUANG, S.; LIU, D.; GAO, G.; GUO, X. A novel method for speckle noise reduction and ship target detection in SAR images. Pattern Recognition, v. 42, p. 1533-1542, 2009.

JENSEN, J. R. Sensoriamento Remoto do Ambiente. Uma Perspectiva em Recursos Terrestres. Upper Saddle River: Prentice Hall, $2^{\mathrm{a}}$ ed., 2009.

LANDIS, J. R.; KOCH, G. G. The measurement of observer agreement for categorical data. Biometrics, v. 33, n. 1, p. 159-174, 1977.

LIU, C.; VACHON, P. W.; GELING, G. W. Improved ship detection with airborne polarimetric SAR data. Canadian Journal of Remote Sensing, v. 31, n. 1, p. 122-131, 2005.

MECOCCI, A.; BENELLI, G.; GARZELLI, A.; BOTTALICO, S. Radar image processing for ship-traffic control. Image and Vision Computing, v. 13, n. 2, p. 119-128, 1995.

MOUCHOT, M. C.; GARELLO, R. SAR for Oceanography. In: HENDERSON, F. M.; LEWIS, A. J. (eds.), Principles \& Applications of Imaging Radar. Manual of Remote Sensing, $3^{\mathrm{a}}$ ed., v. 2, Cap. 12, p. 631-675, 1998. 
PAES, R. L.; LORENZETTI, J. A.; GHERARDI, D. F. M. Ship detection using TerraSAR-X images in the Campos Basin (Brazil). IEEE Geoscience and Remote Sensing Letters, v. 7, n. 3, p. 545-548, 2010.

PARADELLA, W. R.; SANTOS, A.R.; VENEZIANI, P.; CUNHA, E. S. P. Radares imageadores nas Geociências: estado de arte e perspectivas. Revista Brasileira de Cartografia, n. 57/01, p. 56-62, 2005.

RICHARDS, J. A.; JIA, X. Remote Sensing Digital Image Analysis. Nova Iorque: Springer-Verlag, $4^{\mathrm{a}}$ ed. 2006.

ROSA, R. Introdução ao Sensoriamento Remoto. Uberlândia: Universidade Federal de Uberlândia, $3^{\mathrm{a}}$ ed., 1995.

SCHOWENGERDT, R. A. Remote Sensing. Models and Methods for Image Processing. San Diego: Academic Press, $3^{\mathrm{a}}$ ed., 2007.

SILVA, W. F.; RUDORFF, B. F. T.; FORMAGGIO, A. R.; PARADELLA, W. R.; MURA, J. C. Discrimination of agricultural crops in a tropical semi-arid region of Brazil based on L-band polarimetric airborne SAR data. ISPRS Journal of Photogrammetry and Remote Sensing, v. 64, p. 458-463, 2009.

TELLO, M.; LOPEZ-MARTINEZ, C.; MALLORQUI, J. J. Automatic vessel monitoring with single and multidimensional SAR images in the wavelet domain. ISPRS Journal of Photogrammetry \& Remote Sensing, v. 61, p. 260278, 2006.

TOUZI, R.; CHARBONNEAU, F. J.; HAWKINS, R. K.; VACHON, P. W. Ship detection and characterization using polarimetric SAR. Canadian Journal of Remote Sensing, v. 30, n. 3, p. 552-559, 2004.

VIDIGAL, A. A. F.; CUNHA, M. B.; FERNANDES, L. P. C.; MENDES, I. A.; SILVA, N.; OLIVEIRA, L. L.; CUNHA JÚNIOR, O. B.; ALBUQUERQUE, A. T. M.; RODRIGUES, E. G.; ALMEIDA, J. A. N. Amazônia Azul. O Mar que nos Pertence. Rio de Janeiro: Editora Record, 305 p., 2006.

WACKERMAN, C. C.; FRIEDMAN, K. S.; PICHEL, W. G.; CLEMENTECOLON, P.; LI, X. Automatic detection of ships in RADARSAT-1 SAR imagery. Canadian Journal of Remote Sensing, v. 27, p. 568-577, 2001.

WU, G.; LEEUW, J.; SKIDMORE, A. K.; LIU, Y.; PRINS, H. H. T. Performance of Landsat TM in ship detection in turbid waters. International Journal of Applied Earth Observation and Geoinformation, v. 11, p. 54-61, 2009.

(Recebido em Janeiro de 2011. Aceito em setembro de 2011.) 